\title{
Clinical Profile of Stroke-Study Conducted in the Batticaloa Teaching Hospital, Sri Lanka
}

\author{
Maheswaran Umakanth \\ Department of Clinical Sciences, Faculty of Health Care Sciences, Eastern University, Batticaloa, Sri Lanka \\ Email: mumaka nth1972@gmail.com
}

How to cite this paper: Umakanth, M. (2018) Clinical Profile of Stroke-Study Conducted in the Batticaloa Teaching Hospital, Sri Lanka. Journal of Biosciences and Medicines, 6, 1-6.

https://doi.org/10.4236/jbm.2018.66001

Received: May 6, 2018

Accepted: June 3, 2018

Published: June 6, 2018

Copyright $\odot 2018$ by author and Scientific Research Publishing Inc. This work is licensed under the Creative Commons Attribution International License (CC BY 4.0).

http://creativecommons.org/licenses/by/4.0/

\begin{abstract}
Stroke as a disease entity has significantly increased the morbidity, incapacity, and mortality in Sri Lanka. In the west, it is the 3rd most common cause of death. In addition to that, it is a disturbing and restricting cerebrovascular disease with a substantial amount of remaining shortage leading on to emotional and financial burden on the family and society. A hospital-based retrospective study was conducted at Teaching Hospital Batticaloa, Sri Lanka from July 1, 2016, to October 31, 2016. During the study period, data were collected from the medical records. Out of 34 patients, 21 (61.8\%) were males and 13 (38.2\%) were females. The male to female ratio was 1.6:1. Out of 34 patients, 17 (50\%) were affected in the left side and 17 (50\%) were affected on the right side. The most common risk factor was hypertension with $79.4 \%$, and next to that diabetes mellitus (41.2\%), smoking (23.5\%), alcohol (20.6\%), past history of Stroke (17.6\%) and ischaemic heart disease (IHD) (14.7\%). Stroke due to infarction is more common than haemorrhage. In this study, both right and left sides were affected equally. In order to prevent this devastating stroke, acknowledgment of risk factors for stroke is prime importance for the healthcare workers as well as public.
\end{abstract}

\section{Keywords}

Stroke, Stroke in Sri Lanka, Pattern of Stroke

\section{Introduction}

Stroke is a devastating and disabling cerebrovascular disease with a significant amount of residual deficit leading on to emotional and financial burden on the family and society. It has been defined as rapidly developing signs of focal (or global) disturbance of cerebral function with symptoms lasting for more than 24 hours or leading to death with no apparent cause other than vascular origin [1]. 
It is a collection of clinical syndromes resulting from cerebral ischemia to intracranial haemorrhage. In the west, it is the 3rd most common cause of morbidity and mortality. A recent study identified that $7 \%$ of medical and $45 \%$ of neurological admissions were due to stroke with a fatality rate of $9 \%$ at hospital discharge and $20 \%$ at 28 days. Hypertension, alcoholism, smoking, and dyslipidaemia are the commonest cause of stroke among the elderly and however smoking, alcoholism, increased BMI, diabetes, and hypertension are significantly associated with strokes among young people.

Cerebro-vascular accidents occur predominantly in the middle and later years of life and the incidence of stroke increases with age. Thus, disability affects many people in their golden years. Ischemic strokes account for $80 \%-85 \%$ of all strokes worldwide. While Haemorrhagic strokes are due to subarachnoid haemorrhage or intracerebral haemorrhage, they account for $1 \%-7 \%$ and $7 \%-27 \%$ respectively of all strokes worldwide [2]. The American Heart Association/ American Stroke Association (AHA/ASA) estimated the reduction in stroke, coronary heart disease, and cardiovascular risk by $25 \%$ by the year 2010 . The reason for the success was multifactorial and included improved prevention and improved care within the first hours of acute stroke [3].

Patients with acute ischemic stroke, regardless of age or stroke severity, in whom treatment can be started within 3 hours of known onset, should be considered for treatment with alteplase. Patients with primary intracerebral haemorrhage who present within 6 hours of onset with a systolic blood pressure above $150 \mathrm{mmHg}$ should be treated urgently using a locally agreed protocol for blood pressure lowering to a systolic blood pressure of $140 \mathrm{mmHg}$ for at least 7 days, unless the Glasgow Coma Scale score is 5 or less, the haematoma is very large, and death is expected, a structural cause for the haematoma is identified, immediate surgery to evacuate the haematoma is planned [4]. We carried out this study to describe the pattern and clinical profile of stroke at the Teaching Hospital Batticaloa, Sri Lanka which will help young physicians to deal with this deadly \& disabling disease in future.

\section{Material and Methods}

This is a hospital-based retrospective study was conducted at Batticaloa Teaching Hospital, Sri Lanka. For this study, four months period of data were collected from July 1, 2016, to October 31, 2016. A diagnosis of stroke was made according to the World Health Organization(WHO) definition, which defined as interruption of the blood supply to the brain, usually because a blood vessel bursts or is blocked by a clot, causing neurological damages in the means of one side weakness, aphasia, and visual impairment which lasted for more than 24 hours.

During this period, we collected all stroke patient's medical records. We excluded those who had the features of transient ischemic attacks (TIAs). Clinical parameters were collected through a standardized, validated questionnaire from the patient's medical records. Furthermore, full blood count, blood glucose, lipid 
profile, urea, creatinine, electrolytes and a 12 lead ECG finding also were harvested from the medical records. The patients were categorized as infarctions or haemorrhages based on computer tomography of the brain (CT-brain) findings.

In this study, we included 20-years-old or higher with the evidence of stroke. Patients with space-occupying lesions and cortical sinus venous thrombosis were excluded. Risk factors were defined in the following manner. Hypertension was demarcated as previous blood pressure above $140 / 90 \mathrm{~mm} \mathrm{Hg}$ or in the presence of previous specific therapy. Diabetes mellitus was defined as elevated fasting blood glucose above $7 \mathrm{mmol} / \mathrm{l}$ or $\mathrm{HbAlc}>6.5 \%$ or previously on oral hypoglycaemic medication or insulin injection. Dyslipidaemia was defines as total cholesterol above $200 \mathrm{mg} / \mathrm{dl}$, or triglyceride above $150 \mathrm{mg} / \mathrm{dl}$ or low density cholesterol above $160 \mathrm{mg} / \mathrm{dl}$. Those who are smoke any number of cigar or cigarettes for more than one year considered as a risk factor. All data were analysed with the help of Statistical Package for Social Science (SPSS-19).

\section{Results and Discussion}

In the present study, initially, 36 stroke patient's medical records were selected. Two patients were excluded due to inadequate imaging and clinical data. Hence, data from 34 patients were analyzed. Out of 34 patients, 21(61.8\%) were males and $13(38.2 \%)$ were females (Table 1). The male to female ratio was 1.6:1. The incidence of stroke was soaring in the age group of 60 - 69 years which comprises $35.3 \%$ of total patients. Young stroke (age $\leq 50$ years) comprised of $11.8 \%$. Out of 34 patients, 17 (50\%) were affected in in their left side and $17(50 \%)$ were affected in their right side. In this study 29 (85.3\%) were ischemic and 5 (14.7\%) were haemorrhagic type of stroke. Most common risk factor was identified as hypertension (79.4\%), followed by diabetes (41.2\%), smoking (23.5\%), alcohol (20.6\%), past history of Stroke (17.6\%) and IHD (14.7\%) (Table 1).

Among 34 stroke patients, we found a male to female ratio of 1.6 to 1 and this male high proportion was observed in many prior studies across the world [5]. However, high prevalence among male was observed to be the highest in Asia, whereas a lower ratio has been noted in Europe [6]. A similar study was conducted in India where strokes were more common among males (58.53\%) than females (41.46\%) and most common age group was above 60-year-old (58.8\%) [7]. Similar trend was seen in a study conducted by Chirayu V et al [8]. Precisely, a similar sex ratio of 1.6:1 also has been observed in a study completed by Aiyae et al in India [9]. Thus, men are more likely to have a stroke than women [10]. This may be due to differences in risk factors such as smoking and drinking which are more prevalent among men in SriLanka compared with women [11].

The main findings were the significant occurrence of risk factors in our study. Hypertension was documented in 27 patients $(79.4 .2 \%)$ of the study population. Other risk factors in descending order were diabetes 14 (41.2\%), smoking 8 (23.5\%), alcohol 7 (20.6\%), past history of stroke $6(17.6 \%)$, ischemic heart disease 5 (14.7\%) and dyslipidaemia 3 (8.82\%). Hypertension was a common risk factor with $32 \%$ to $65 \%$ of the ischemic stroke. This pattern was seen in a study 
Table 1. Clinical profile of stroke.

\begin{tabular}{|c|c|c|}
\hline & Frequency & Percentage \\
\hline \multicolumn{3}{|l|}{ Gender } \\
\hline Male & 21 & $61.8 \%$ \\
\hline Female & 13 & $38.2 \%$ \\
\hline Total & 34 & $100 \%$ \\
\hline \multicolumn{3}{|c|}{ Stroke in relation between age group } \\
\hline $20-39$ & 2 & $5.9 \%$ \\
\hline $40-49$ & 2 & $5.9 \%$ \\
\hline $50-59$ & 10 & $29.4 \%$ \\
\hline $60-69$ & 12 & $35.3 \%$ \\
\hline More than 70 & 8 & $23.5 \%$ \\
\hline Total & 34 & $100 \%$ \\
\hline \multicolumn{3}{|l|}{ Side of the weakness } \\
\hline Left & 17 & $50 \%$ \\
\hline Right & 17 & $50 \%$ \\
\hline Total & 34 & $100 \%$ \\
\hline \multicolumn{3}{|l|}{ Type of the Stroke Patients } \\
\hline Ischemic & 29 & $85.3 \%$ \\
\hline Haemorrhagic & 05 & $14.7 \%$ \\
\hline Total & 34 & $100 \%$ \\
\hline \multicolumn{3}{|l|}{ Risk factors among stroke } \\
\hline Hypertension & 27 & $79.4 \%$ \\
\hline Smoking & 8 & $23.5 \%$ \\
\hline Diabetes mellitus & 14 & $41.2 \%$ \\
\hline Dyslipidaemia & 3 & $8.82 \%$ \\
\hline Past history of stroke & 6 & $17.6 \%$ \\
\hline Ischaemic Heart Disease & 5 & $14.7 \%$ \\
\hline Alcohol & 7 & $20.6 \%$ \\
\hline
\end{tabular}

conducted by Divyant R in 2016 [12]. Hypertension as a risk factor was also noted more commonly in Asian studies [6] [13]. However, in this study nearly $80 \%$ of population among stroke is hypertension. This percentage is higher than available literature mentioned here.

The second most risk factor in this study was diabetes. The prevalence of diabetes is increasing in Sri Lanka which ranges from 19\% to 21\%. Around 1.5 million Sri Lankan adults do suffer from diabetes and the number is expected to rise up to 2.1 million by the year 2030. Diabetes mellitus is the leading cause of myocardial infarction, stroke, chronic kidney disease, blindness in adults and 
amputations [14]. The prevalence of diabetes varied widely between studies in the Asian literature, ranging from $7.3 \%$ to $52.2 \%$ [15]. The prevalence of smoking in our study was $23.5 \%$ which was lower than that of several Western studies where prevalence ranges from $44 \%$ to $47 \%$, but higher than that of previous Asian studies [7] [16] [17].

An abnormal lipid profile is found to be significantly associated with the occurrence of ischemic stroke. The study findings are supported by various studies in the literature in an Asian country. However, in this study, the prevalence of dyslipidaemia was 3 (8.82\%). Interestingly similar pattern was observed in India, where a study conducted by Sanjay V, et al. [7]. Furthermore, a similar study conducted in north part of the Sri Lanka where prevalence was 15.8\% [13] In present study percentage of ischemic heart disease, smoking and alcohol were less or more same as compared to other studies. In this study, most common type of stroke was cerebral infarction (85.3\%), and second most common type of stroke was haemorrhagic (14.7\%). It correlates with a study conducted by Chirayu $\mathrm{V}$ et al, in which infarction was $85.3 \%$. A similar trend was observed in most of the Asian countries [12] [18].

\section{Limitations of the Study}

We initially, plan to collect more sample, unfortunately we had a problem with our CT-machine. This technical problem narrowed sample size. As this was the retrospective study, we mainly depend on the patient's medical records.

\section{Conclusion}

Stroke is still a major problem and the major predisposing factor remains uncontrolled hypertension. Men are more likely to have a stroke than women. Though majority of case $(84.9 \%)$ were seen in the elderly, stroke in the young carries special importance as they form the most productive age group. Acknowledgement of risk factors for stroke is prime importance for the health care workers as well as public.

\section{Consent for Publication}

Consent was obtained from the director, Teaching Hospital Batticaloa for publication of this article.

\section{Availability of Data and Material}

All data gathered during this study are included in this published article.

\section{References}

[1] Foundation, S. (2017) Clinical Guidelines for Stroke Management Summary of Recommendations. Clinical Guidelines for Stroke Management Summ Recomm.

[2] Feigin, V., Lawes, C., Bennet, D. and Barker Cello, S.P.V. (2009) Worldwide Stroke Incidence and Early Case Fatality in 56 Population Based Studies. The Lancet Neurology, 8, 355-369. https://doi.org/10.1016/S1474-4422(09)70025-0 
[3] Adams, H.P., Bruno, A., Connors, J.J.B., et al. (2013) AHA/ASA Guideline Guidelines for the Early Management of Patients With Acute Ischemic Stroke. $A H A / A S A$ Guidel.

[4] Party, S.W. (2016) National Clinical Guideline for Stroke. Royal College of Physicians.

[5] Kwon, S.U., Kim, J.S., Lee, J.H., et al. (2000) Ischemic Stroke in Korean Young Adults. Acta Neurologica Scandinavica, 101, 19-24. https://doi.org/10.1034/j.1600-0404.2000.00004.x

[6] Spengos, K. (2010) VKR Factors. Etiology and Outcome of First-Ever Ischemic Stroke in Young Adults Aged 15-45. European Journal of Neurology, 17, 1358-1364. https://doi.org/10.1111/j.1468-1331.2010.03065.x

[7] Patne, S.V. and Chintale, K.N. (2016) Study of Clinical Profile of Stroke Patients in Rural Tertiary Health Care Centre. International Journal of Advances in Medicine, 3, 666-670.

[8] Vaidya, C.V. and Majmudar, D.K. (2014) A Study on Clinical Profile of Stroke in Young and Elderly in GMERS Medical College and Hospital, Gandhinagar, Gujarat. International Journal of Research in Medical Sciences, 2, 1446-1452.

[9] Aiyar, I. (1999) A Study of Clinic-Radiological Correlation in Cerebrovascular Stroke. Gujarat Medical Journal, 52, 58-63.

[10] Jadhav, Y.L. and Bondarde, S.A. (2015) Study of Risk Factors and Clinical Profile of Stroke in Young Adults. MVP Journal of Medical Sciences, 2, 15-19. https://doi.org/10.15306/mvpjms/2015/v2i1/58607

[11] Das, S.B.T. (2008) Stroke Indian Scenario. Circulation, 118, 2719-2724. https://doi.org/10.1161/CIRCULATIONAHA.107.743237

[12] Divyant, R. (2016) STROKE : The Study of Clinical Profile and Risk Factor in Tertiary Care Hospital. Rawal Divyant Varshney Amit Medical Science. International Journal of Science and Research, 82, 187-188.

[13] Arasalingam, A., Kumanan, T., Kamalakkannan, K. and Visvakumar, M. (2016) Stroke Subtypes and Risk Factors in the Jaffna District-A Hospital Based Study Based Study. Sri Lanka Journal of Neurology.

[14] Somasundaram, N.P., Wijeyaratne, C.N., De Silva, S., et al. (2013) Diabetes Mellitus: Glucose Control Clinical guidelines: The Endocrine Society of Sri Lanka. Sri Lanka Journal of Diabetes Endocrinology and Metabolism, 453, 45-57. https://doi.org/10.4038/sjdem.v3i1.5505

[15] Ramachandran, A., Snehalatha, C. and Shetty, A.S. (2012) Trends in Prevalence of Diabetes in Asian Countries. World Journal of Diabetes, 3, 110-117. https://doi.org/10.4239/wjd.v3.i6.110

[16] Varona, J.F., Guerra, J.M., Bermejo, F., et al. (2007) Causes of Ischemic Stroke in Young Adults, and Evolution of the Etiological Diagnosis over the Long Term. European Neurology, 57, 212-218. https://doi.org/10.1159/000099161

[17] Kwon, S.U., Kim, J.S., Lee, J.H., et al. (2000) Ischemic Stroke in Korean Young Adults. Acta Neurologica Scandinavica, 101, 19-24. https://doi.org/10.1034/j.1600-0404.2000.00004.x

[18] Eapen, R., et al. (2009) A Study of Clinical Profile and Risk Factors of Crerbrovascular Strok. Gujarat Medical Journal, 64, 47-54. 